\title{
EEG Dipole Source Localization using Deep Neural Network
}

\author{
Zeng Hui ${ }^{1}$, Li Ying ${ }^{1,2}$, Wang Lingyue ${ }^{1}$, Yin Ning ${ }^{1,2}$, Yang Shuo ${ }^{1,2}$ \\ 1. (Tianjin Key Laboratory of Bioelectromagnetic Technology and Intelligent Health, Hebei University \\ of Technology, Tianjin 300130, China) \\ 2. (State Key Laboratory of Reliability and Intelligence of Electrical Equipment, Hebei University of \\ Technology, Tianjin 300130, China) \\ Corresponding author: Li Ying, Email: yli@hebut.edu.cn
}

Received: April 10, 2021. Revised: December 20, 2021. Accepted: January 5, 2022. Published: January 7, 2022.

\begin{abstract}
Electroencephalography (EEG) inverse problem is a typical inverse problem, in which the electrical activity within the brain is reconstructed based on EEG data collected from the scalp electrodes. In this paper, the four-layer concentric head model is used for simulation firstly, four deep neural network models including a multilayer perceptron (MLP) model and three convolutional neural networks (CNNs) are adopted to solve EEG inverse problem based on equal current dipole (ECD) model. In the simulations, 100,000 samples are generated randomly, of which $60 \%$ are used for network training and $20 \%$ are used for cross-validation. Eventually, the generalization performance of the model using the optimal function is measured by the errors in the rest $20 \%$ testing set. The experimental results show that the absolute error, relative error, mean positioning error and standard deviation of the four models are extremely low. The CNN with 6 convolutional layers and 3 pooling layers $(\mathrm{CNN}-3)$ is the best model. Its absolute error is about 0.015 , its relative error is about 0.005 , and its dipole position error is $0.040 \pm 0.029 \mathrm{~cm}$. Furthermore, we use $\mathrm{CNN}-3$ for source localization of the real EEG data in Working Memory. The results are in accord with physiological experience. The deep neural network method in our study needs fewer calculation parameters, takes less time, and has better positioning results.
\end{abstract}

Keywords-inverse problem, EEG, DNN, source localization.

\section{INTRODUCTION}

$\mathrm{E}$ LECTROENCEPHALOGRAPHY (EEG) inverse problem aims at reconstructing the source information of electrical activity inside the brain based on the electrical signals measured on the scalp, and it is widely used in both clinical diagnosis and cognitive function research of brain because of its advantages of high speed, safety, and noninvasiveness [1,2]. The source localization is a typical inverse problem. Due to the ill-posed and underdetermined character of the problem, EEG inverse problem has no unique solution, i.e., a measured electric field can be explained by infinite numbers of different source configurations possibly. Some geometrical and mathematical constraints must be added to gain the most reasonable solution[3]. Usually, equivalent current dipole (ECD) methods and imaging methods are used to describe the activity of the brain. We discuss the method to localize the current sources within the brain with ECD model in this paper.

When the electrical activities within small regions of the brain are simulated as ECDs, the task of EEG inverse problem is to find the optimal parameter estimation (position and polar moment) of ECDs $[4,5]$. The optimization algorithm must be used to find the optimal solution. Some local optimization algorithm and global optimization methods, such as least square method, clustering method, simulated annealing, genetic algorithm, etc., are proposed for EEG source localization [6].

Neural networks, especially the feedforward neural networks, have strong abilities to approximate complex nonlinear mapping functions [7]. In the early research, shallow neural network methods, such as artificial neural network (ANN), the combination of wavelet and neural network (WNN), and support vector machine (SVM), have been used in the EEG source location [8-12]. Shallow neural network could learn the intrinsic functional relationship between EEG and brain-source generators and extract shallow simple feature information, but complex information is difficult to reflect by shallow network. The accuracy of its positioning results is significantly lower than that of traditional fitting methods [13].

Deep neural network (DNN) makes a breakthrough in multiple fields in recent years [14]. According to the universal approximation theorem, a shallow feedforward network can approximate any measurable function, provided the network is given enough hidden units. But its layer may be infeasible large and may fail to learn correctly. However, the experience of predecessors shows that if the number of model parameters increases but the network depth doesn't change, the increase of improvement will be limited [15]. Further, deeper networks need far fewer parameters, and can generalize frequently. Therefore, the deep network is more suitable for complex function approximation than the shallow model.

Researchers have applied DNN methods to process 
biomedical data. For example, EEG signal classification, medical image analysis and so on. Many pieces of research show that DNNs can deal with the ill-posed inverse problem and source localization problem[16-18]. Some studies have shown that it is helpful to deal with ill-posed inverse problems [19]. And convolutional neural networks were used for ill-posed inverse problems in 12-lead ECG[20]. The recursive neural network of long short-term memory (LSTM) has also been applied to solve process of inverse EEG problems and achieved effective results[21]. In the case of noise, it has better robustness compared with the traditional dipole fitting method.

Applying neural networks with the advantages of self-learning, associative storage and fast calculations to inverse problem solving can build memory during training and correctly infer the intrinsic functional relationship between the scalp EEG and the endogenous position of the brain, and find the inverse solution online. Compared with the shallow network used in earlier studies, the DNN is more feasible for representing the complex mapping relationship between electrical signals and source power activity, and the accuracy of the results is also higher.

In this paper, four DDNs models, including a multilayer perceptron (MLP) model and three convolutional neural network $(\mathrm{CNN})$ models, are constructed to solve EEG inverse problem based on ECD model, and reconstruction results are compared and analyzed. To find the best DNN model of positioning effect, it is used to locate the source of EEG signal. The results illustrate the superior quality of DNN methods in EEG source localization.

\section{THE GENERAL FORM OF EEG INVERSE PROBLEM}

Due to the high concentration and sparseness of the brain electrical activities, the local source in the brain can be modeled as a single current dipole with the parameter $(\boldsymbol{r}, \boldsymbol{q})$, where, $\boldsymbol{r}=\left(r_{x}, r_{y}, r_{z}\right)$ is the position vector and $\boldsymbol{q}=\left(q_{x}, q_{y}, q_{z}\right)$ is the moment vector [22]. The relationship between the scalp potential $u$ on electrodes $m$ and the dipole source can be simplified into a transmission system. If the noise on $m$ potential points is $N$, the relationship between the scalp potential and the single dipole source can be expressed as:

$\boldsymbol{u}=L(\boldsymbol{r}, \boldsymbol{q})+\boldsymbol{N}$

where $\boldsymbol{u}=\left[u_{1}, u_{2}, \cdots, u_{m}\right]^{\mathrm{T}}$ is the potential distribution on $m$ points, and the nonlinear operator $L$ is the transfer function reflecting the inherent characteristics of the system depending on the conductivity characteristics of the model, the grid division, and the electrodes distribution on the measurement space, etc.

EEG inverse problem is a nonlinear optimization problem, and the main idea is to fit the forward calculated potential distribution with the measured scalp data by adjusting the parameters of the dipole. The mathematic form can be expressed to the minimum of the objective function based on the least-square error $\min _{\boldsymbol{r}, \boldsymbol{q}} e(\boldsymbol{r}, \boldsymbol{q})$. The objective function is as follows.

$$
g(\boldsymbol{r}, \boldsymbol{q})=\|\boldsymbol{u}-\tilde{\boldsymbol{u}}\|_{2}^{2}=\sum_{i=1}^{m}(\boldsymbol{u}-\tilde{\boldsymbol{u}})^{2}
$$

where $\tilde{\boldsymbol{u}}$ is the measured potential distribution on the scalp.

The EEG source location problem has become a multi-parameter optimization problem. Generally, the optimization method is studied to find the parameters that minimize the objective function. However, due to the ill-posed problem, the parameter optimization process is difficult to complete. It is necessary to introduce various constraints in the solution process, and then find a suitable approximate solution through various numerical calculation criteria.

\section{THEORY OF DEEP NEURAL NETWORK}

\section{A. Multilayer perceptron}

Multilayer perceptron (MLP) is a common and relatively simple feedforward neural network, which is generally composed of input layer, hidden layer, and output layer. When MLP is used to accept input $x$ and produce output $y$, information flows forward through the network. The input $x$ provides the initial information that then propagates up to the hidden units at each layer and finally produces $y$ which is a nonlinear weighted sum of input. This process is called forward propagation, which is illustrated as follows.

$$
\left\{\begin{array}{c}
\boldsymbol{a}^{0}=\boldsymbol{x} \\
\boldsymbol{z}^{j}=\boldsymbol{W}^{j} \boldsymbol{a}^{j-1}+\boldsymbol{b}^{j} \\
\boldsymbol{a}^{j}=f\left(\boldsymbol{z}^{j}\right) \\
\boldsymbol{y}=\boldsymbol{a}^{l}
\end{array}\right.
$$

where, $\boldsymbol{a}^{0}$ is initial information, $\boldsymbol{a}^{j}$ is the output of the $j$-th layer, $\boldsymbol{z}$ is the input of the hidden layer, $\boldsymbol{W}$ is the weight matrix, $\boldsymbol{b}$ is the bias vector, $j=(0,1, \ldots, l)$ is the number of layers in the network, and $f(\cdot)$ called activation function is a non-linear function.

When constructing MLP, we need to determine some details. Typically, a loss function is defined to measure the performance of the model. Then, an activation function is specified. Non-linearity of the neural network is achieved by using affine transformation followed by an activation function. The affine transformation is controlled by learned parameters including weights $\boldsymbol{W}$ and the biases $\boldsymbol{b}$. After that, we select an optimization method to update parameters. In addition, other hyper-parameters, such as architecture and regularization, affect the performance of the model. These details are described below.

\section{1) Loss function}

Common loss functions of neural networks include cross entropy loss, L1 loss, L2 loss, etc. The result of the regression task is an integer or a real number, so the common loss functions of regression task have L1 loss, L2 loss, and their improvements. Usually, the data in the task is normalized, the sample data is less than 1. Some simulation results show that L2 loss is not particularly robust. When the predicted value of the 
function is far from the true value, it may cause the gradient to explode. By contrast, L1 loss function is not easily affected by the large error. It is also called mean absolute error (MAE), and the function can be defined as follows.

$$
M A E=\frac{1}{k} \sum_{i=1}^{k}\left|y_{i}-\tilde{y}_{i}\right|
$$

where, $m$ is the number of samples. $y_{i}$ is the predicted value, and $\tilde{y}_{i}$ is the true value.

2) Activation function

The activation functions commonly used in neural networks include sigmoid function, tanh function, rectified linear unit function (ReLU), etc. Some studies show that sigmoid and tanh functions have the problem of gradient disappearance. The ReLU function can effectively alleviate this problem, which has the advantages of easy optimization, avoiding gradient explosion problems, and simplifying the calculation process. Despite these considerable advantages, the ReLU function is no longer working when the input is less than zero. However, the leaky ReLU function uses a non-zero slope when $x<0$, which makes the activation function meaningful in the negative domain [23] . The leaky ReLU can be defined as follows.

Leaky ReLU $=\left\{\begin{array}{c}x, x>0 \\ \lambda x, x<0\end{array}\right.$

where $\lambda \in(0,1)$ is a constant.

3) Optimization method for network parameters

Neural networks are usually trained by using iterative gradient-based optimizer that drives the loss function to an extremely low value. The backpropagation (BP) algorithm allows the information from the loss to through the network to compute the gradient. After these gradients have been computed, the optimization algorithm that we selected uses these gradients to update the parameters of this network.

Common optimization algorithms include stochastic gradient descent, mini-batch gradient descent, adaptive moment estimation (Adam), and so on [24] . Adam algorithm can calculate the adaptive learning rate of each parameter, avoiding the problem that the learning rate is difficult to choose in the gradient descent method. Compared with other adaptive learning rate algorithms, its convergence speed is faster, and the learning is more effective.

4) Selection of architecture

The architecture refers to the overall structure of the network. Its main considerations are choosing the depth of the network and the width of each layer. The ideal network architecture for a task must be found via experimentation guided by monitoring the validation set error.

5) Regularization

Regularization, which can prevent the model from overfitting, is widely used in model training. Common regularization methods are the early stopping, the L1/L2 weight decay and the dropout method. The early stopping method is designed to solve the problem that the number of epochs needs to be manually set.
After each epoch, the test results of the verification set and record the best verification set accuracy so far. As the epoch increases, if the test error is found to increase on the verification set, then stop training. The weight of the highest accuracy rate in the previous processing of the test set is used as the final parameter of the network.

\section{(B. Convolutional neural network}

Convolutional neural network $(\mathrm{CNN})$ is one of the most successful cases of DNN. It is a special deep feedforward neural network designed under the influence of the concept of "receptive field" in the field of biological neuroscience [25]. The traditional CNN structure consists of input layer, convolution layer, pooling layer, fully connected layer, and output layer. The convolution layer and the pooling layer are unique structures of CNN.

The convolutional layer repeatedly acts on each receptive field of the entire input signal through a convolution kernel, and the result of the convolution constitutes a feature map of the input signal. Assuming that the input of the 1-th convolutional layer is represented by $z^{l}$ and the output is represented by $a^{l}$, the relationship between the output and the input is defined as follows.

$\left\{\begin{array}{c}\boldsymbol{a}^{l}=f\left(\boldsymbol{z}^{l}\right) \\ \boldsymbol{z}^{l}=\sum_{i=1}^{M} \boldsymbol{a}_{i}^{l-1} * \boldsymbol{k}_{i}^{l}+\boldsymbol{b}^{l}\end{array}\right.$

where $f(\cdot)$ is the activation function, $M$ is the number of convolution kernel, $\boldsymbol{k}$ is the convolution kernel matrix, $\boldsymbol{b}$ is the bias parameter, and $*$ is the convolution symbol.

The purpose of the pooling layer is to compress the input matrix, reduce the amount of network calculations, and make the model more robust. The specific operation is to divide the input feature map into multiple non-overlapping areas, and then compress each region according to the pooling standard, which is generally the average value or the maximum value, which ensures the invariance of feature translation and scaling. The symbol pool $(\bullet)$ represents the pooling function. Then the output of layer $l+1$ is defined as follows.

$\boldsymbol{a}^{l+1}=\operatorname{pool}\left(\boldsymbol{a}^{l}\right)$

After multiple alternating convolution and pooling layers, the next is the fully connected layer, which expands all the feature maps into one-dimensional features as the input of the fully connected network. After the weighted summation and activation function of the previous layer, the output of the fully connected $m$-layer can be obtained, as shown in equation (3).

The CNN training algorithm is also mainly based on the BP algorithm of gradient descent. The goal is to estimate network parameters based on training samples and expected output, including convolution kernel parameter $k$, fully connected layer network weight $\boldsymbol{W}$ and each layer bias $\boldsymbol{b}$.

\section{EEG inverse problem using deep neural network}

EEG dipole source location problem is expressed to get a set of $(\boldsymbol{r}, \boldsymbol{q})$ (dipole parameters) given a set of input $\boldsymbol{u}$ (scalp 
potential) through a certain state transition.

To solve the dipole parameters by using the neural network method, many training samples are obtained by the forward calculation of EEG firstly. Secondly, the neural network is trained by the training set. After that, the optimal function is used to minimize the loss function by adjusting the hyper-parameters and cross verifying the training results on the validation set. Finally, the trained model is adopted to predict source information through the the potential distribution on the scalp surface, and the optimal function on the test set was selected. The whole process is shown in Figure1.

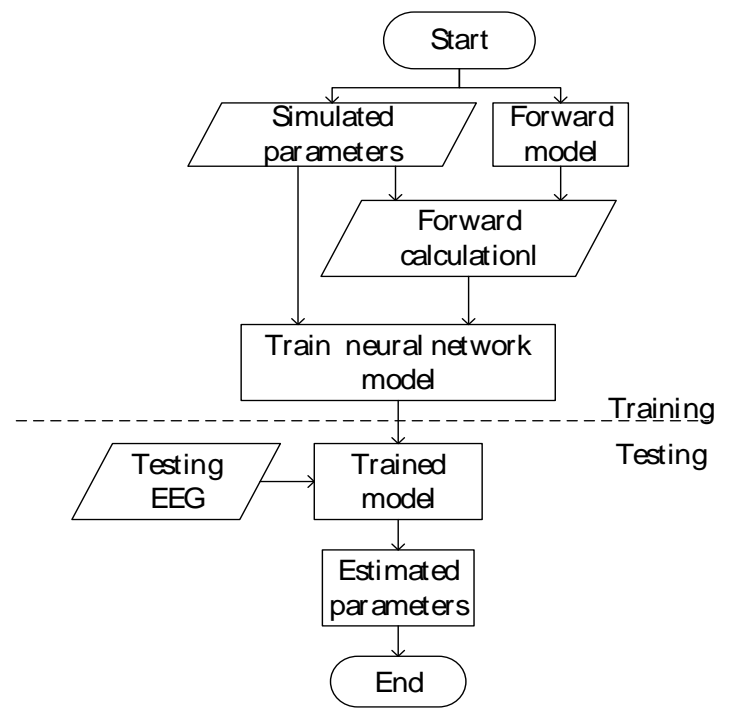

Fig.1 Network flowchart

\section{RESULTS}

\section{A. Generating data using forward model}

To solve the EEG inverse problem, it is necessary to determine the geometric model of the head and the impedance distribution information of each part. Some studies have shown that using neural network methods, high-precision real head model takes more time and computational cost to perform forward calculations, but the source location accuracy is not significantly improved [8]. Furthermore, to simplify the calculation and save the computation cost, 4-layer concentric sphere model is chosen as the head model, which considers the inhomogeneity of the conductivity of each layer of the head [27]. A 4-layer concentric sphere model was established as shown in Figure.2. It includes brain, cerebrospinal fluid, skull, and scalp from inside to outside. The electrical conductivities of each part are $0.33 \mathrm{~S} \bullet \mathrm{m}-1,1 \mathrm{~S} \bullet \mathrm{m}^{-1}, 0.0042 \mathrm{~S} \bullet \mathrm{m}^{-1}$, and 0.33 $\mathrm{S} \bullet \mathrm{m}^{-1}$, the relative radii are $0.8,0.85,0.92$, and 1 , respectively, and the radius $R$ of the outermost scalp is $9 \mathrm{~cm}$.

In this study, the electrode placement of EEG signal is 128 channels, which in accordance with the international 10-20 electrode lead positioning standard. The result of the forward problem is the analytical solution. Using the method of reference [26], the result which includes the corresponding scalp potential of the dipole source is approximated through the
Legendre polynomial transformed into a closed-form formula.

It is generally believed that the dipole source can be located at any positions in the cerebral cortex, and the location of the dipole source is randomly selected. In the 4-layer concentric sphere model, the three coordinates of the dipole position vector $\boldsymbol{r}$ are sampled independently with an equal probability distribution. The three components of the dipole moment $\boldsymbol{q}$ are also randomly generated on a normal distribution with zero mean and unit variance. For each dipole, the moment is different.

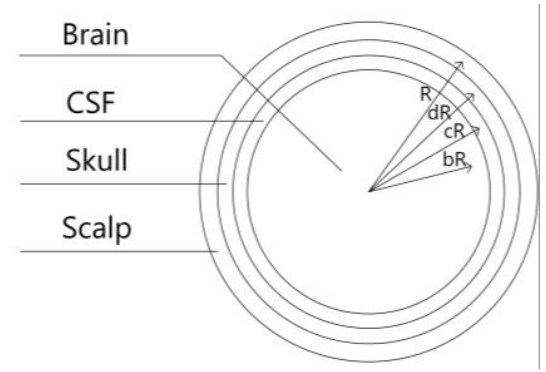

Fig.2 Four-layer concentric model

\section{B. Modeling with deep neural networks}

In this study, we establish four network models with different structures including one MLP model and three CNN models.

Firstly, we built a fully connected MLP structure. After some testing, the best performing MLP architecture is selected. There are seven layers, including six hidden layers and one output layer. The size of the layers is 768, 512, 320, 256, 128, 6 from hidden layer to output layer. The Leaky ReLU written in equation (5) is selected as the activation function of the model.

Then, we built a simple structure of CNN, containing only one convolutional layer and one maximum pooling layer $(\mathrm{CNN}-1)$. The convolutional layer has $3 \times 3$ filters, and the number of filters is 32. The Leaky ReLU is used as the activation function of the convolutional layer.

Furthermore, we built the third model named CNN-2. It contains 6 convolutional layers and 6 pooling layers, i.e., the structure of $\mathrm{CNN}-1$ repeats five times. The convolutional layers have $3 \times 3$ filters, and the number of filters is $32,32,64,64$, 128,128 in sequence. The other hyper-parameters are the same as CNN-1.

The fourth model (CNN-3) is a structure similar in Visual Geometry Group (VGG) network, which has small convolution kernel and good generalization performance. CNN-3 has 6 convolutional layers and 3 pooling layers, i.e., the structure with two convolutional layer and one maximum pooling layer repeats three times. The other hyper-parameters are the same as CNN-2.

All max-pooling is implemented for $2 \times 2$ patches. After all CNN structures, 3 fully connected layers containing 512, 256, and 6 hidden units are attached to obtain the output. The total parameters of MLP, CNN-1, CNN-2, and CNN-3 are about 5M, $1 \mathrm{M}, 0.7 \mathrm{M}$, and $0.5 \mathrm{M}$, respectively.

For comparing the differences between models, the same hyper-parameters should be selected in the design of models. The loss function chose the MAE in equation (4), and Adam is 
selected as the optimization algorithm. In additional, batch normalization $(\mathrm{BN})$ layer is added to the networks for preventing overfitting. We also examined more complex CNN structures, but they did not show obvious improvement in the localization performance.

\section{Experiment with simulation data}

The simulated signal is used for the experiment to show the performance of the proposed approach. A total of 100,000 sets of analog signals are generated, $60 \%$ of which are used as the training set, $20 \%$ as the validation set, and $20 \%$ as the test set. Each signal is 128 channels located by the standard BIOSEMI-128 EEG system. The four networks are trained on the training set with 400 epochs. For each network, the loss functions on validation sets are shown in Figure 3. CNNs converge around 50 epochs, whereas MLP converges after 250 epochs. For MLP, CNN-1, and CNN-2 models, the loss function on the verification set drops to about 0.03 after 400 iterations. It can be seen from the figures that the loss function of $\mathrm{CNN}-3$ is the lowest.

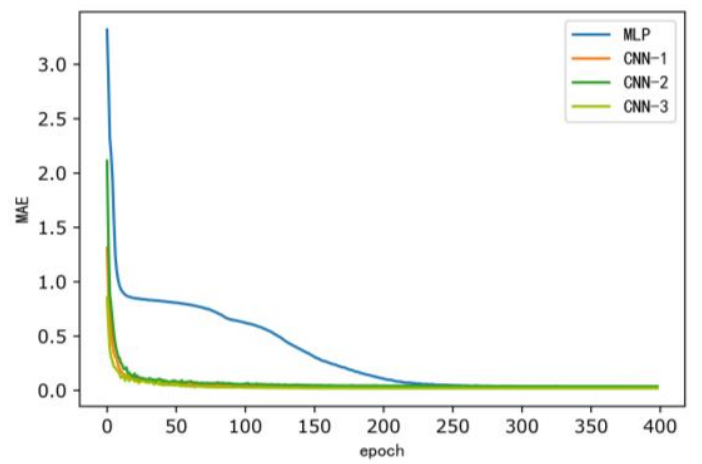

(a)

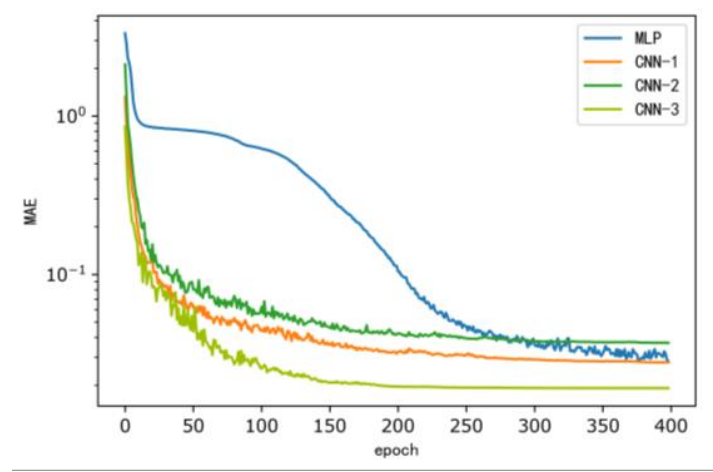

(b)

Fig.3 Performance of four models. (a) shows the loss function MAE, and (b) is its logarithmic graph

The early stop method is used to find the optimal function in training. The loss function changes as shown in Figure 4, where only the model of error reduction is recorded. After several iterations, the loss curves converge to a low level. Meanwhile, the network shows good generalization on the validation set.

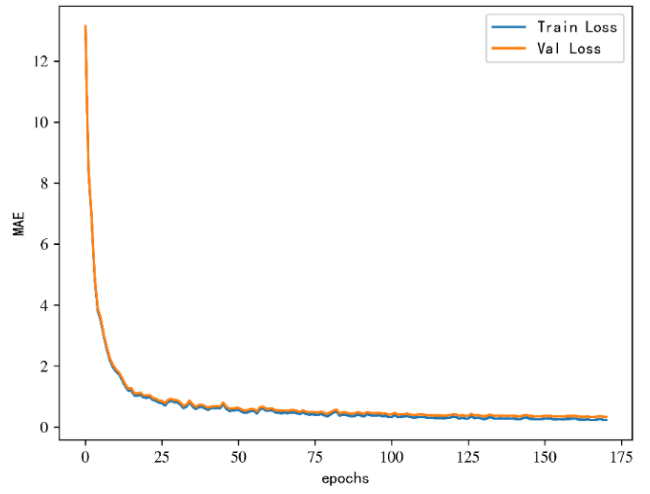

Fig.4 Performance of model based on early stopping

The proposed approach estimates the source location with dipole parameter regression. After selecting optimal function, the performance of four models is evaluated by using errors between estimated parameter and ground-truth parameter in the simulated data. To quantitatively evaluate the result of dipole source positioning, the the global and individual errors of absolute error (Aerror) and relative error (Rerror) are calculated, respectively. The formulas are defined as follows.

$$
\begin{gathered}
\text { Aerror }=\frac{\sum_{i=1}^{N} \sum_{r=1}^{S}\left|y_{i r}-\tilde{y}_{i r}\right|}{S N} \\
\text { Rerror }=\sqrt{\frac{\sum_{i=1}^{N} \sum_{r=1}^{S}\left|y_{i r}-\tilde{y}_{i r}\right|^{2}}{\sum_{i=1}^{N} \sum_{r=1}^{S} y_{i r}{ }^{2}}}
\end{gathered}
$$

where $S$ is the number of components in the output vector, $N$ is the number of samples, $\tilde{y}$ is the actual value, and $y$ is the predicted value.

Calculating the overall error of the six parameters of a dipole from equation (8) and equation (9), and then calculating the errors of the six parameters separately to avoid interference between data changes of different magnitudes. The error of the test set is shown in Table 1 and Table 2. Compared with other methods, the Aerror and Rerror of CNN-3 are the smallest. According to the comparison of calculation cost and localization error of six parameters, $\mathrm{CNN}-3$ is the best performing model. Its total positioning errors of the six parameters using are the smallest, and the estimation of each parameter is also satisfactory.

For further measuring the positioning error, the mean localization error distance of dipole positioning is calculated. The formulas are defined as follows.

$D=\|\boldsymbol{r}-\tilde{\boldsymbol{r}}\|$

$\bar{r}=\frac{1}{N} \sum_{i=1}^{N} D_{i}$

$S D=\sqrt{\frac{1}{N} \sum_{i=1}^{N}\left(D_{i}-\bar{r}\right)^{2}}$

where, $\tilde{\boldsymbol{r}}$ is the actual value of position vector, and $\boldsymbol{r}$ is the predicted value. $D$ is the Euclidean distance, $\bar{r}$ is mean error distance and $S D$ is the standard deviation. 
Table 1 Localization Aerror of dipole

\begin{tabular}{|c|c|c|c|c|}
\hline Method & MLP & CNN-1 & CNN-2 & CNN-3 \\
\hline$(\boldsymbol{r}, \boldsymbol{q})$ & 0.0282 & 0.0350 & 0.0249 & 0.0148 \\
\hline$r_{x}$ & 0.0416 & 0.0357 & 0.0283 & 0.0207 \\
\hline$r_{y}$ & 0.0413 & 0.0388 & 0.0289 & 0.0273 \\
\hline$r_{z}$ & 0.0377 & 0.0303 & 0.0183 & 0.0167 \\
\hline$q_{x}$ & 0.0231 & 0.0378 & 0.0167 & 0.0083 \\
\hline$q_{y}$ & 0.0222 & 0.0400 & 0.0188 & 0.0085 \\
\hline$q_{z}$ & 0.0234 & 0.0277 & 0.0175 & 0.0121 \\
\hline
\end{tabular}

Table 2 Localization Rerror of dipole

\begin{tabular}{|c|c|c|c|c|}
\hline Method & MLP & CNN-1 & CNN-2 & CNN-3 \\
\hline$(\boldsymbol{r}, \boldsymbol{q})$ & 0.0091 & 0.0122 & 0.0123 & 0.0050 \\
\hline rx & 0.0205 & 0.0219 & 0.0246 & 0.0191 \\
\hline ry & 0.0306 & 0.0273 & 0.0262 & 0.0169 \\
\hline rz & 0.0178 & 0.0133 & 0.0124 & 0.0073 \\
\hline qx & 0.0112 & 0.0125 & 0.0122 & 0.0024 \\
\hline qy & 0.0091 & 0.0133 & 0.0140 & 0.0025 \\
\hline qz & 0.0050 & 0.0058 & 0.0054 & 0.0025 \\
\hline
\end{tabular}

After calculation, the experimental results $\bar{r}$ and SD of the four networks are recorded in Table 3. The positioning distances of neural network methods are generally low, between $0.8 \sim 0.4 \mathrm{~mm}$. Their positioning error is lower than the artificial neural network model [8]. It is observed that the total parameters and train time of $\mathrm{CNN}-3$ are the smallest, while $\mathrm{CNN}-2$ is the second smallest among the four models. Among them, CNN-3 achieved the best positioning effect, and the optimal result of the test set is $0.398 \pm 0.286 \mathrm{~mm}$. Therefore, CNN-3 is used as the source location model in the following paragraphs.

Table 3 Comparison of position results

\begin{tabular}{|c|c|c|c|}
\hline \multirow{2}{*}{ Method } & \multirow{2}{*}{ Times $(s)$} & \multicolumn{2}{|c|}{ The position error $(\mathrm{cm})$} \\
\cline { 3 - 4 } & & $\bar{r}$ & $S D$ \\
\hline MLP & 5330.48 & 0.0824 & \pm 0.0782 \\
\hline CNN-1 & 3014.14 & 0.0703 & \pm 0.0661 \\
\hline CNN-2 & 6737.37 & 0.0502 & \pm 0.0425 \\
\hline CNN-3 & 6529.66 & 0.0398 & \pm 0.0286 \\
\hline
\end{tabular}

To visually perform localization results and generalization capabilities of the CNN-3 model, randomly select 100 samples from the new test set and compare the difference between the predicted values of the 6 dipole parameters and the true values. From Figure 5, the red hollow circles represent the predicted values, and the green points represent the true values. Among them, the red circles cover the green points well, which means the fitting results of the dipole position parameters and the dipole moment parameters are awfully close true values, and indicates the positioning effect of the deep neural network is good.
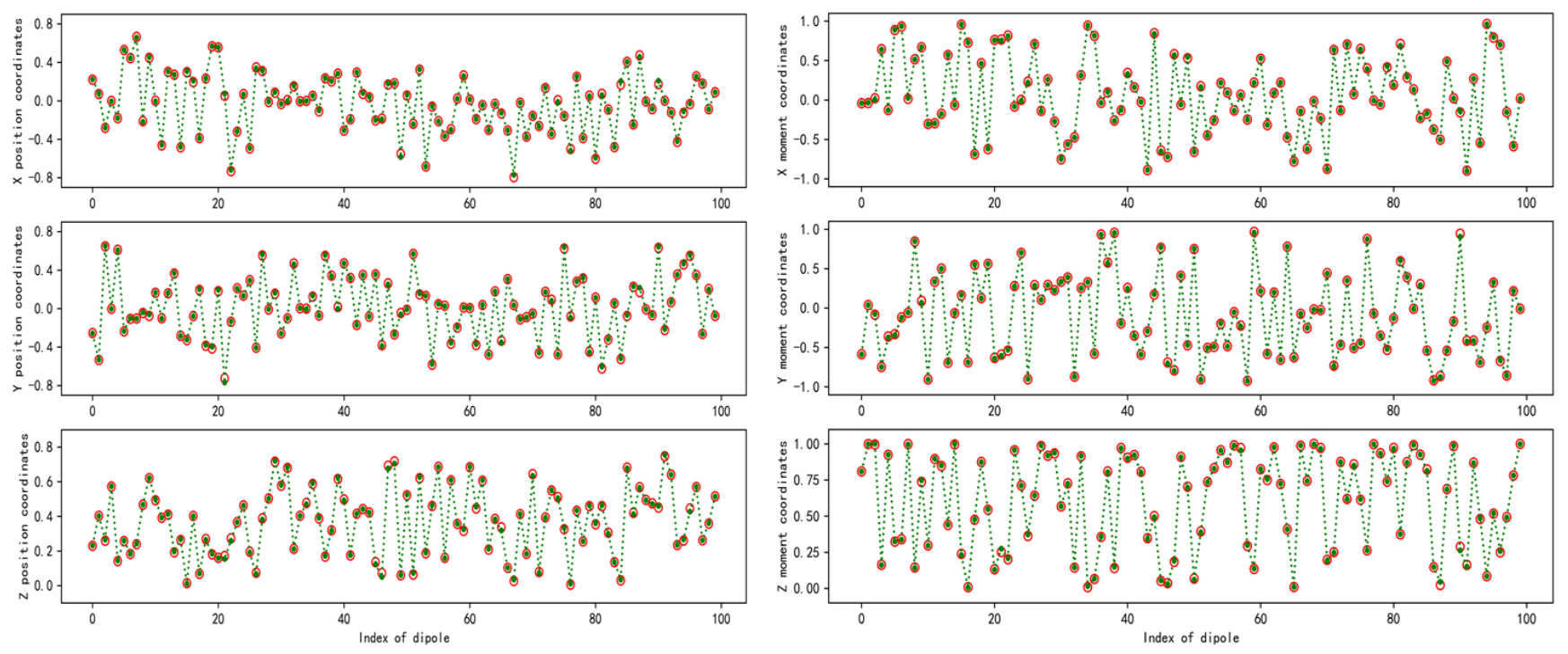

Fig.5 Result of 100 single dipole localization using CNN-3

\section{Experiment with real EEG data in working memory}

EEG is a helpful tool to study the cognition function of the brain such as working memory. Working memory refers to the ability to store and maintain pertinent information (e.g., short-term memory), as well as applying the information with the help of higher-level executive processes. It is currently one of the most active research fields in cognitive neuroscience.

We use the Deese-Roediger-McDermott (DRM) paradigm to study the effect of emotion on working memory. The improved DRM paradigm working memory task experiment was designed, and 28 subjects were selected to participate in the experiment. The experimental materials include 12 emotional word lists mixed with 4 positive, 4 neutral, and 4 negative. Each word list contains 12 learning words (all of them appear in the learning phase, some of them appear in the test phase) and 1 keyword (it only appears in the test phase). In the learning phase, the lists were mixed and presented to the participants, and each word was displayed on the computer monitor for $1 \mathrm{~s}$. During the test phase, each word was displayed on the computer monitor for $2 \mathrm{~s}$. The participants need to judge whether they have seen the word in the learning phase by pressing different keys on the keyboard. Neuroscan equipment is used to collect 
64 channel EEG signals of subjects in remembering Chinese words with different emotions. The distribution of electrode channels conforms to the 10-20 electrode lead positioning standard.

The obtained EEG is pre-processed to remove kinds of noise and artifact. The peak point after preprocessing is selected as the time point. The CNN-3 model is trained using 64-pilot simulation EEG data. Its hyper-parameters are the same as the simulation model. 60,000 samples are used to train. Its training takes $4728.53 \mathrm{~s}$. After that, the trained network is used to locate 24 points of the real EEG in three emotions. It takes about $2 \mathrm{~s}$.

The source localization results of different brain regions are selected for mapping. The results of the positive group are shown in Figure 6. Figure 6(a) and Figure 6(b) indicate the source is mainly activated in the left prefrontal lobe and the temporal lobe. The source localization results of the neutral group are shown in Figure 7. Figure 7(a) and Figure 7(b) indicate the source is mainly activated in the posterior frontal lobe and the temporal lobe. The source localization results of the negative group are shown in Figure 8. Figure 8(a) and Figure 8 (b) indicate the source is primarily activated in the prefrontal lobe and the temporal lobe.

The activation region of the estimated source is mainly located in the frontal lobe and temporal lobe. It is consistent with the source localization results by using commercial software Curry 8, which proves the effectiveness of the proposed method on real EEG signals.

The positioning results of the three emotions can be seen from the activation position that the activation position of the frontal lobe of the subjects in the positive and negative emotional states is biased to the frontal lobe, but the activation source of the positive group is biased to the left hemisphere of the brain, and the activation source of the negative group is biased. In the right hemisphere, the main activation area of the calm group is more inclined to the posterior frontal and temporal lobes. The left and right hemispheres of the human brain play different roles. Among them, the left hemisphere of the brain processes cognitive activities such as language, reading and reasoning. The right hemisphere of the brain is mainly responsible for cognitive functions such as space and music, and the prefrontal lobes of the left and right hemispheres are involved in the regulation of emotions [27]. Memory items in this experiment are words rather than objects, are related to verbal working memory rather than object working memory [28]. The localization results are consistent with physiological knowledge related to working memory [29, 30].

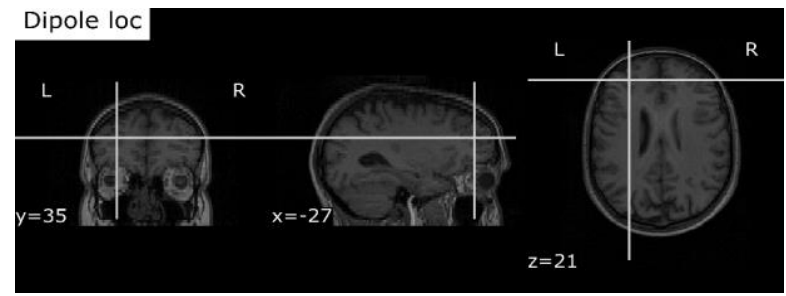

(a)

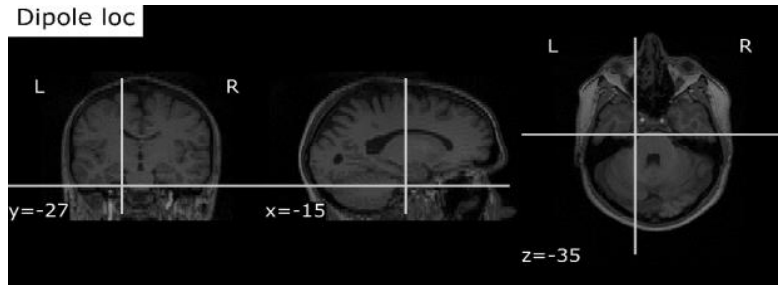

(b)

Fig.6 Source localization result of the positive group

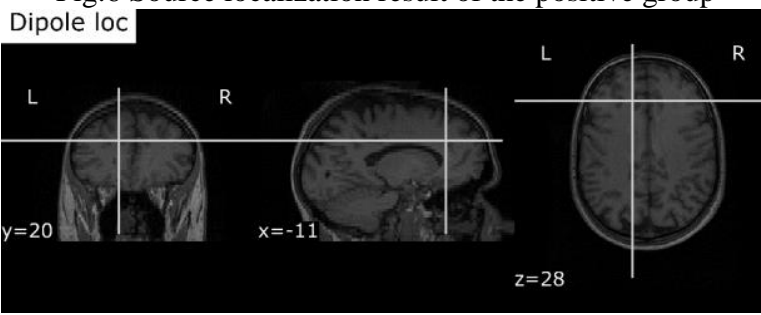

(a)

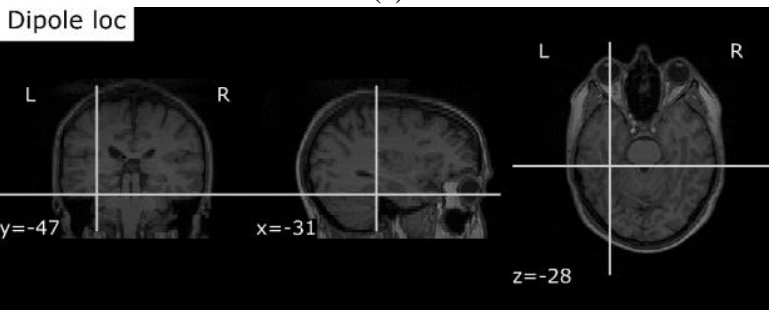

(b)

Fig.7 Source localization result of the neutral group

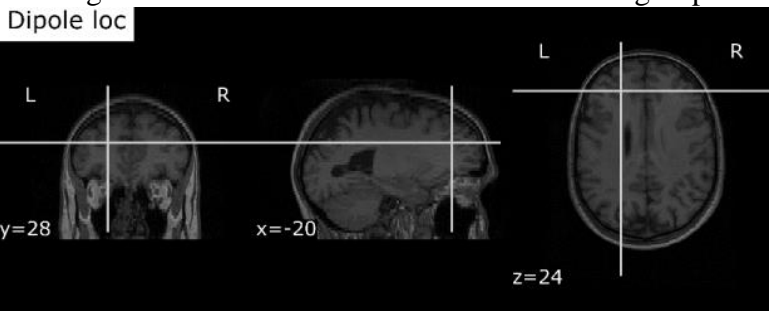

(a)

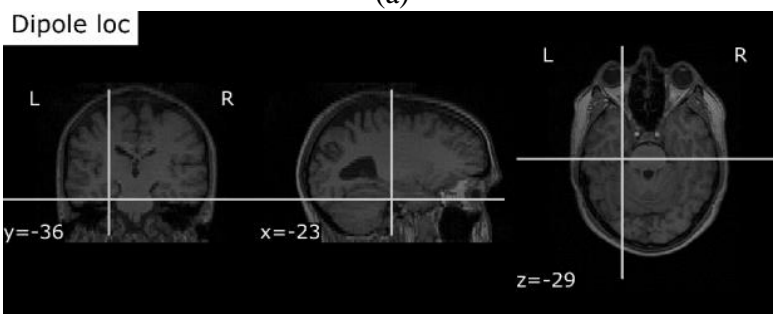

(b)

Fig. 8 Source localization result of the negative group

\section{DISCUSSION}

In this study, four DNNs with different structures are established to locate the EEG dipole source. Through the analysis of the positioning results of the four models, it is found that the errors are incredibly low, which proves the effectiveness of MLP and CNN in the process of solving the EEG inverse problem. Compared with the shallow model, the deep models have a better approximation on the EEG inverse problem and higher positioning accuracy.

CNN-1 and MLP have no obvious difference in positioning accuracy. However, $\mathrm{CNN}-1$ model has fewer parameters than 
MLP. It indicates that shallow CNN and fully connected networks have similar results in the application of dipole positioning, but the CNN-1 model is more efficient than MLP. Furthermore, the deeper $\mathrm{CNN}-2$ and $\mathrm{CNN}-3$ spent more time and have higher positioning accuracy than $\mathrm{CNN}-1$. It proves the usability of the deeper $\mathrm{CNN}$ in this field.

For the four networks, CNN-3 is the most prominent model. Compared with other networks, its total parameter and calculation cost are the smallest, and the calculation accuracy is the highest. In terms of structure, $\mathrm{CNN}-3$ includes more convolutional layers, so it has a stronger ability to express data features. In contrast, $\mathrm{CNN}-2$ loses some information through the dimension reduction in the pooling layer. Its accuracy is lower than $\mathrm{CNN}-3$.

During the calculation of the inverse problem, the effect of the pooling layer is not obvious because of the small data, but the full convolution structure can achieve higher accuracy on this occasion. The forecast time of four models for the new dipole parameters is about $5 \mathrm{~s}$. And these models achieve high positioning accuracy and avoid the problem of long calculation time of traditional methods. The results show the effectiveness and superiority of the DNN method, especially CNN, in EEG inverse problem. And CNN-3 is the superior model among them.

When CNN-3 is used to locate the source of real EEG signals in working memory, the sources of EEG with three emotions are mainly activated in the prefrontal lobe and the temporal lobe. It is in accordance with the source localization results of software Curry, and consistent with the physiological knowledge related to working memory. These results reveal the proposed method is acceptable on EEG inverse problem of real signals.

Convolutional neural network is used to directly estimate source parameters. It can more accurately approximate the complex conduction process of neural signals and accurately locate local sources. And through the learning ability of neural networks, the source can be quickly located, which can better utilize the high time resolution of EEG.

\section{CONCLUSIONS}

In this paper, four DNN models including a MLP model and three CNNs are proposed to solve EEG inverse problem based on ECD model. The simulation results for four-layer concentric head model demonstrate that DNNs can obtain high positioning accuracy localization and can generalize frequently. Also, they can speed up the calculation process of inverse problems without given prior knowledge.

When DNN is applied to locate the source of real EEG data in working memory experiments, the results are consistent with physiological knowledge. These outcomes demonstrate the proposed DNN methods are beneficial to advance the research of the EEG source localization problem and helpful to accelerate the study of brain function.

\section{References}

[1] C.M. Michel, B. He, "EEG source localization," Handbook of Clinical Neurology, vol. 160, pp. 85-101 2019.

[2] M.A. Jatoi, N. Kamel, A.S. Malik, I. Faye, T. Begum, "A survey of methods used for source localization using EEG signals," Biomedical Signal Processing and Control, vol. 11, pp. 42-52 2014.

[3] K. Wendel, O. Vaisanen, J. Malmivuo, N.G. Gencer, B. Vanrumste, P.J. Durka, et al., "EEG/MEG source imaging: methods, challenges, and open issues," Computational Intelligence and Neuroscience, vol. 2009, pp. 1-12 2009.

[4] Y. Li, H. Li, R. He, L. Rao, Q. Wu, G. Xu, et al., "EEG source localization using differential evolution method," International Conference of the IEEE Engineering in Medicine and Biology Society, vol. 1, pp. 1903-1906 2004.

[5] C.M. Michel, M.M. Murray, G. Lantz, S.L. Gonzalez, L. Spinelli, R.G. De Peralta, "EEG source imaging," Clinical Neurophysiology, vol. 115, pp. 2195-2222 2004.

[6] D. Escalonavargas, D. Gutierrez, I. Lopezarevalo, "Performance of different metaheuristics in EEG source localization compared to the Cramér-Rao bound," Neurocomputing, vol. 120, pp. 597-609 2013.

[7] L.C. Jiao, S.Y. Yang, F. Liu, S.G. Wang, Z.X. Feng, "Seventy years beyond neural networks: retrospect and prospect," Chinese Journal of Computers, vol. 39, pp. 1697-1716 2016.

[8] G. Van Hoey, J. De Clercq, B. Vanrumste, R.V. De Walle, I. Lemahieu, M. Dhave, et al., "EEG dipole source localization using artificial neural networks," Physics in Medicine Biology, vol. 45, pp. 997-1011 2000.

[9] K. Kamijo, T. Kiyuna, Y. Takaki, A. Kenmochi, T. Tanigawa, T. Yamazaki, "Integrated approach of an artificial neural network and numerical analysis to multiple equivalent current dipole source localization," Frontiers of Medical Biological Engineering, vol. 10, pp. 285-301 2000.

[10] R.J. Sclabassi, M. Sonmez, M. Sun, "EEG source localization: a neural network approach," Neurological Research, vol. 23, pp. 457-464 2001.

[11] L.S. Qing Wu, Youxi Wu, Guizhi Xu, Ying Li, Weili Yan, "Combining nonlinear dimensionality reduction with wavelet network to solve EEG inverse problem," International Conference of the IEEE Engineering in Medicine and Biology Society, vol. 2006, pp. 4245-4248 2006.

[12] Y.-H.W. Jian-Wei Li, Gui-Long Zong, Qing Wu, "Support vector machine method using in EEG signals study of epileptic spike," International Conference on Machine Learning and Cybernetics vol. 2, pp. 1241-1245 2009.

[13] M. Bianchini, F. Scarselli, "On the complexity of neural network classifiers: a comparison between 
shallow and deep architectures," IEEE Transactions on Neural Networks, vol. 25, pp. 1553-1565 2014.

[14] K. He, X. Zhang, S. Ren, J. Sun, "Deep residual learning for image recognition," 2016 IEEE Conference on Computer Vision and Pattern Recognition (CVPR), pp. 770-778 2016.

[15] I. Goodfellow, Y. Bengio, A. Courville, "Deep learning," MA: The MIT Press, pp. 2016.

[16] N. Yalta, K. Nakadai, T. Ogata, "Sound source localization using deep learning models," Journal of Robotics and Mechatronics, vol. 29, pp. 37-48 2017.

[17] K.H. Jin, M.T. Mccann, E. Froustey, M. Unser, "Deep convolutional neural network for inverse problems in imaging," IEEE Transactions on Image Processing, vol. 26, pp. 4509-4522 2017.

[18] A. Lucas, M. Iliadis, R. Molina, A.K. Katsaggelos, "Using deep neural networks for inverse problems in imaging: beyond analytical methods," IEEE Signal Processing Magazine, vol. 35, pp. 20-36 2018.

[19] J. Adler, O. Oktem, "Solving ill-posed inverse problems using iterative deep neural networks," Inverse Problems, vol. 33, pp. 1240072017.

[20] T. Yang, L. Yu, Q. Jin, L. Wu, B. He, "Localization of origins of premature ventricular contraction by means of convolutional neural network from 12-lead ECG," IEEE Transactions on Biomedical Engineering, vol. 65, pp. 1662-1671 2018.

[21] S. Cui, L. Duan, B. Gong, Y. Qiao, F. Xu, J. Chen, et al., "EEG source localization using spatio-temporal neural network," China Communications, vol. 16, pp. 131-143 2019.

[22] S.K. Veeramalla, V.K.H.R. Talari, "Multiple dipole source localization of EEG measurements using particle filter with partial stratified resampling," Biomedical Engineering Letters, vol. 2020, pp. 1-11 2020.

[23] G. Wang, G.B. Giannakis, J. Chen, "Learning ReLU networks on linearly separable data: algorithm, optimality, and generalization," IEEE Transactions on Signal Processing, vol. 67, pp. 2357-2370 2019.

[24] D.P. Kingma, J. Ba, "Adam: a method for stochastic optimization," International Conference on Learning Representations 2015.

[25] C. Bo, P. G, S. G, "Deep learning with hierarchical convolutional factor analysis," IEEE Trans Pattern Anal Mach Intell, vol. 35, pp. 1887-1901 2013.

[26] M. Sun, "An efficient algorithm for computing multishell spherical volume conductor models in EEG dipole source localization," IEEE Transactions on Biomedical Engineering, vol. 44, pp. 1243-1252 1997. A. Baddeley, "Working memory: theories, models, and controversies," Annual Review of Psychology, vol. 63, pp. 1-29 2006.

[28] M.E. Thomason, E. Race, B. Burrows, S. Whitfield-Gabrieli, G.H. Glover, J.D.E. Gabrieli, "Development of spatial and verbal working memory capacity in the human brain," Journal of Cognitive Neuroscience vol. 21, pp. 316-332 2009.

[29] L. Wang, X. Liu, K.G. Guise, R.T. Knight, J.J.J.o.C.N. Fan, "Effective connectivity of the fronto-parietal network during attentional control," Journal of Cognitive Neuroscience, vol. 22, pp. 543-553 2009.

[30] C. Elfgren, D.V. Westen, U. Passant, E.M. Larsson, P. Mannfolk, P.J.N. Fransson, "fMRI activity in the medial temporal lobe during famous face processing," NeuroImage, vol. 30, pp. 609-616 2006.

\section{Ethics approval and consent to participate :}

The experimental data in this study were obtained with the informed consent of all participants. The institutional review board of the Hebei University of Technology approved the experiment, code HEBUThMEC2020006.

\section{Contribution of Individual Authors to the Creation of a Scientific Article (Ghostwriting Policy)}

Zeng Hui: Investigation, Writing - original draft.

Li Ying: Conceptualization, Methodology, Writing review \& editing.

Wang Lingyue: Software, Writing - review \& editing.

Yin Ning: Supervision, Validation.

Yang Shuo: Supervision, Validation.

\section{Creative Commons Attribution License 4.0} (Attribution 4.0 International, CC BY 4.0)

This article is published under the terms of the Creative Commons Attribution License 4.0 https://creativecommons.org/licenses/by/4.0/deed.en_US 